\title{
Degradation of Road Tested Automotive Connectors
}

\author{
Johnathan Swingler, John W. McBride, and Christian Maul
}

\begin{abstract}
The automotive environment is particularly demanding on connector performance, and is characterized by large temperature changes, high humidity and corrosive atmospheres. This paper presents an initial study of connector performance in terms of temperature profiles taken from road vehicles. The temperature profiles are then simulated using empirical relationships to allow prediction of connector performance.

Wire harnesses have been investigated to seek evidence of the connector degradation predicted from the temperature data. Initial indications are that the wire harness shows the type of fretting behavior associated with the temperature changes. Evidence of fretting corrosion was found at the contact interface on tin plated terminals from sealed and unsealed connectors.
\end{abstract}

Index Terms-Automotive connectors, degradation mechanisms, operating environments.

\section{INTRODUCTION}

I $\mathrm{N}$ TERMS of reliability the connector is often a weak link in electrical and electronic systems used in road vehicles. The increasing complexity of systems leads to increased numbers of connectors, with the need to reduce packaging space. A high specification vehicle can have in excess of 400 connectors with 3000 individual terminals [1]. Field data has shown that between $30-60 \%$ of electrical failures are attributed to connector problems [2], [3]. A typical failure is defined as a high contact resistance or intermittent contact resistance faults [4], [5]. The degradation mechanisms, which lead to a high contact resistance, have been extensively investigated over many years; this has resulted in accelerated simulated tests to aid in connector design and verification of performance [6]-[11]. Recent studies have shown that synergistic relationships between the degradation mechanisms can lead to more complex failure modes than previously assumed [2].

The aim of this study is to collect data on environmental conditions, which will lead to a better understanding of connector failure mechanisms. The study includes data collection of temperature, humidity and vibration related to specific connectors on vehicles under test in the United Kingdom, Canada, and the United States. The United Kingdom data is for temperate conditions, while the data collected in the USA is for hot arid conditions, and in Canada cold winter conditions. In this paper initial results are presented on the temperature characteristic of connectors on a road vehicle in the United Kingdom. This paper focuses upon several features exhibited in the temperature profile of a selection of connectors. An empirical temperature model

Manuscript received October 1, 1999; revised December 10, 1999. This paper was recommended for publication by Associate Editor M. Braunovic upon evaluation of the reviewers' comments.

The authors are with University of Southampton, Southampton S017 1BJ, U.K.

Publisher Item Identifier S 1521-3331(00)02249-2. is developed to generalize the features observed, and the rate constants are discussed. Following this an analysis of sample connectors is presented showing the degradation mechanisms associated with these temperature features.

\section{Methodology}

The connectors in a motor vehicle will experience a number of operational stresses from the external and local environment. In this paper the emphasis is on temperature data. The stresses the connector can experience are:

Temperature: The automotive connector is usually classified in one of several high temperature operating regimes, e.g.: 85 , 105,125 , and $155^{\circ} \mathrm{C}$ [12].

Corrosive Gas Attack: The most common corrosive gas is oxygen leading to the formation of undesired oxides. Other gases include $\mathrm{SO}_{2}, \mathrm{NO}_{2} \mathrm{Cl}_{2}$, and $\mathrm{H}_{2} \mathrm{~S}$.

Humidity: Corrosion is exacerbated by humidity where water can condense to form galvanic cells. Mechanical wear of a contact surface has been reported to be influenced by humidity levels [13].

Vibration: Sources of vibration include the engine, gearbox, power train and vibrations transmitted through the suspension.

\section{A. Temperature Measurements}

The connector temperature was measured with standard K-type thermocouples. The thermocouples were attached to the connector housing facing the engine, since this is where the highest temperatures are expected. The signals were sampled every $90 \mathrm{~s}$.

The temperature profiles of three types of connector are considered in this paper. These connectors are taken from a number of positions in the engine compartment of a vehicle where a variety of temperature levels can be identified. The types of connector include

- low temperature $\left(85^{\circ} \mathrm{C}\right)$ sealed connector mounted on the body;

- medium temperature $\left(105^{\circ} \mathrm{C}\right)$ sealed connector mounted on the body;

- high temperature $\left(125^{\circ} \mathrm{C}\right)$ sealed connector mounded on the engine.

\section{TEMPERATURE RESUltS}

A considerable amount of data has been acquired typified by the profile shown in Fig. 1. This figure shows a snapshot of temperature data collected from one connector as the vehicle was driven following a test procedure lasting several days, in the UK. Two 4-day tests and one 15-day test were carried out. The temperature was monitored continually for the whole test with 


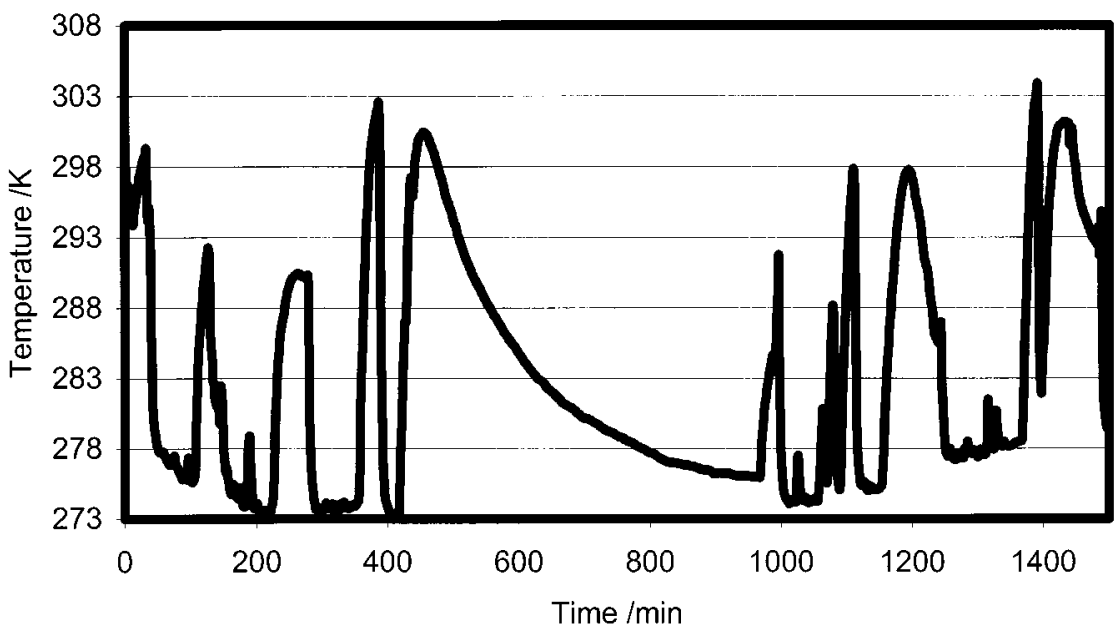

Fig. 1. Typical acquired experimental data.

a sample rate of $90 \mathrm{~s}$. The test procedure consisted of several elements: warm up while stationary, driving at specific speeds for set time periods, letting the vehicle stand stationary while the engine ran for set time periods, and turning off and letting the engine cool. The example data in Fig. 1 shows results from a single connector taken over a $24-\mathrm{h}$ period. The figure shows there are sudden increases and decreases in temperature from 273 to $303 \mathrm{~K}$. The slow decrease in temperature indicated in the middle of the graph corresponds to the engine being turned off. The rapid changes in the temperature data correspond to conditions where the vehicle is stationary for a period (heating up) before moving off (cooling down).

It was found that the connector temperature was a function of several parameters such as vehicle velocity, the speed of the engine (RPM), whether the cooling fan had been activated, connector type and its position under the bonnet.

Data collected from vehicles in Canada and the USA show that for the Type III connector, the highest temperature was similar in all environments. The further away the connector from the engine the more significant the influence of the ambient temperature, [14].

\section{TEMPERATURE MODEL}

The objective of the model is to give a temperature profile of an automotive connector at particular scenarios for a limited number of input parameters. The empirical temperature model was developed to generalize features in the data by using a minimal number of these parameters. The features of interest are:

1) rapid increase in temperature on vehicle start-up (thermal shock);

2) highest temperature achieved;

3) the fast changes in temperature when the vehicle is traveling;

4) slow cooling when vehicle is at rest.

The expressions used in the model are based on an exponential first-order rate law [15] of the form,

$$
T(t)=T_{\infty}+\left(T_{0}-T_{\infty}\right) \exp (-K t)
$$

TABLE I

MODEL COEFFICIENTS

\begin{tabular}{l|c|c|c|c}
\hline Type & $\mathrm{T}_{\mathrm{amb}} / \mathrm{K}$ & $\Delta \mathrm{T}_{\mathbf{h}} / \mathbf{K}$ & $\mathbf{K}_{\mathbf{h}} / \mathbf{m i n}^{-1}$ & $\mathbf{r}$ \\
\hline I & 273 & 27.5 & 0.1 & 0.986 \\
\hline II & 273 & 41 & 0.1 & 0.964 \\
\hline III & 273 & 64 & 0.1 & 0.885 \\
\hline
\end{tabular}

where

$T(t)$ connector temperature at time $t$ into a scenario;

$T_{0} \quad$ temperature at the start of that scenario;

$T_{\infty} \quad$ final temperature of the scenario;

$K$ rate constant.

The model is developed for three scenarios, where the inputs are limited to temperature parameters and a rate constant. A current loading term is not included in the temperature model since in all cases the thermocouples are mounted on the housing surface, and therefore primarily reflect the local ambient temperature.

\section{A. Stationary Vehicle with a Connector Heating-Up}

The experimental data show that the sample connectors heated-up to a maximum temperature dependent upon the connector type and position. The temperature change $\left(\Delta T_{h}\right)$ for the heat up part of the cycle, is used in (2) to model the behavior using the values shown in Table I. $\Delta T_{h}$ and $T_{a m b}$ values were taken from the experimental data. The profiles, $T_{h}(t)$, were found by fitting the best curve to the experimental data so as to maximize the correlation coefficient, $r$. Three sets of experimental data were used for each type of connector

$$
T_{h}(t)=T_{a m b}+\left(\Delta T_{h}-\Delta T_{h} \exp \left(-K_{h} t\right)\right) .
$$

Fig. 2 shows heat-up profiles for the three types of connector. Experimental data and empirically modeled curves are shown. The experimental data is shown to drift slightly with connector type III and this is reflected in the correlation coefficient in 


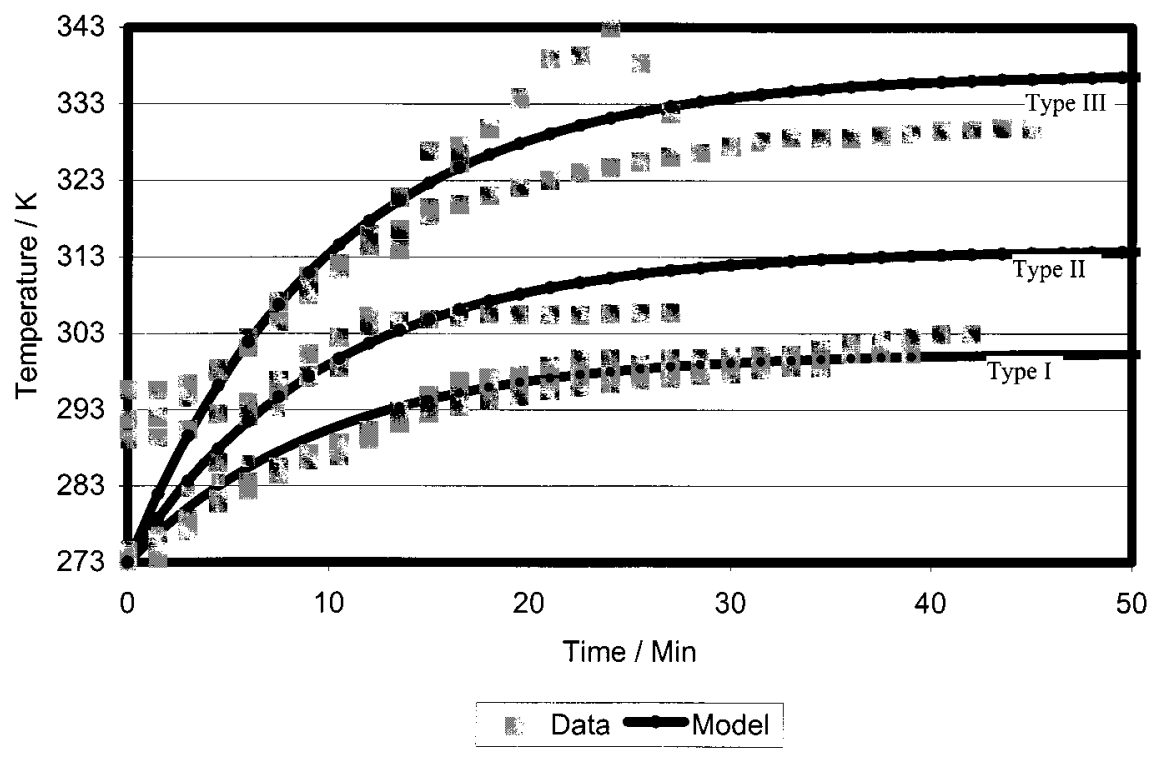

Fig. 2. Heat-up temperature profile connectors.

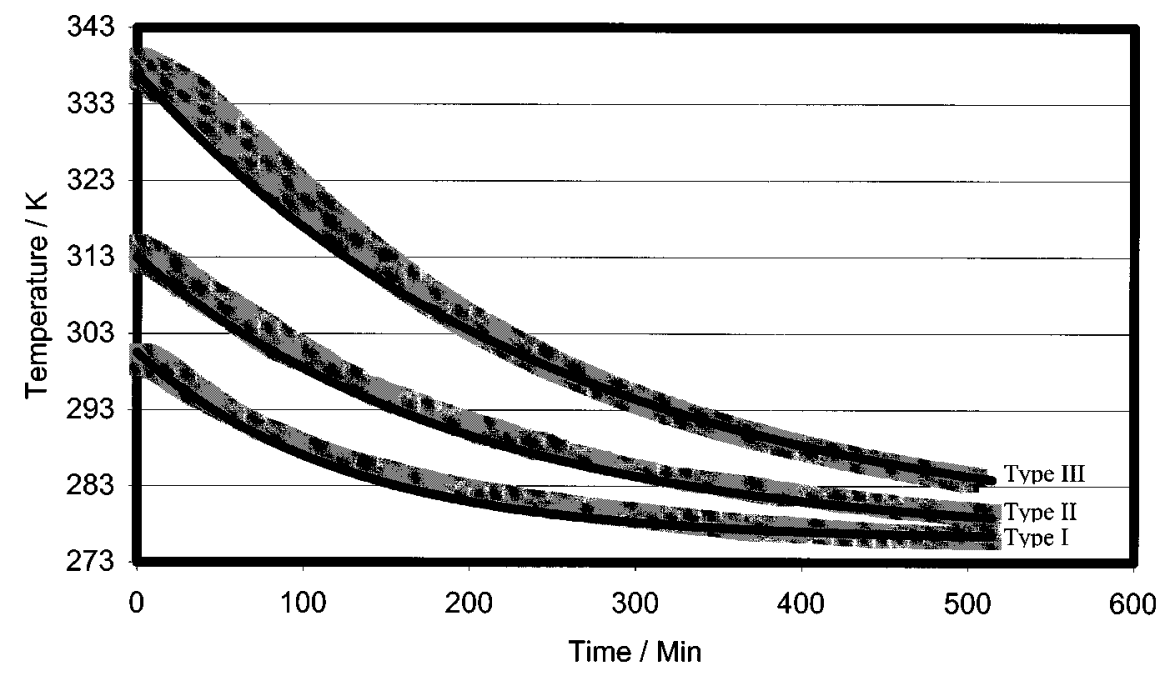

Data Model

Fig. 3. Cool-down temperature profile of connectors.

Table I. It is interesting to note that in all cases $\mathrm{K}$ is the same for the three terminal types.

\section{B. Stationary Vehicle with Connector Cooling-Down}

Fig. 3 shows three sets of experimental data for each connector type. The grey area corresponds to a large number of data points from the experimental data. The decrease in temperature $\left(\Delta T_{h}\right)$ is slower relative to the heat-up. Three curves are plotted of the model using (3) and the coefficients in Table II. These can be seen to have a high correlation to the experimental data. $\Delta T_{h}$ and $T_{a m b}$ values were taken from the experimental data

$$
T_{c}(t)=T_{a m b}+\Delta T_{h} \exp \left(-K_{c} t\right) .
$$

It can be seen from Fig. 3 that after $8 \mathrm{~h}$ (500 min) the connectors are still cooling and above ambient temperature.
TABLE II

MODEL COEFFIEIENTS

\begin{tabular}{l|c|c|c|c}
\hline Type & $\mathbf{T}_{\mathbf{a m b}} / \mathbf{K}$ & $\Delta \mathbf{T}_{\mathbf{h}} / \mathbf{K}$ & $\mathbf{K}_{\mathrm{c}} / \mathbf{m i n}^{-1}$ & $\mathbf{r}$ \\
\hline I & 276 & 24.5 & 0.008 & 0.998 \\
\hline II & 276 & 37 & 0.005 & 0.999 \\
\hline III & 276 & 61 & 0.004 & 0.998 \\
\hline
\end{tabular}

\section{Traveling Vehicle with Connector Cooling-Down}

Duringb vehicle travel the temperature profile was found to be dependent upon a number of parameters. However, a high and low temperature value was found for each connector type during travel, $T_{a m b}+\Delta T_{h}$ and $T_{a m b}+\Delta T_{l}$. Modeling cooling from high to low temperature was achieved by modifying (3) without 


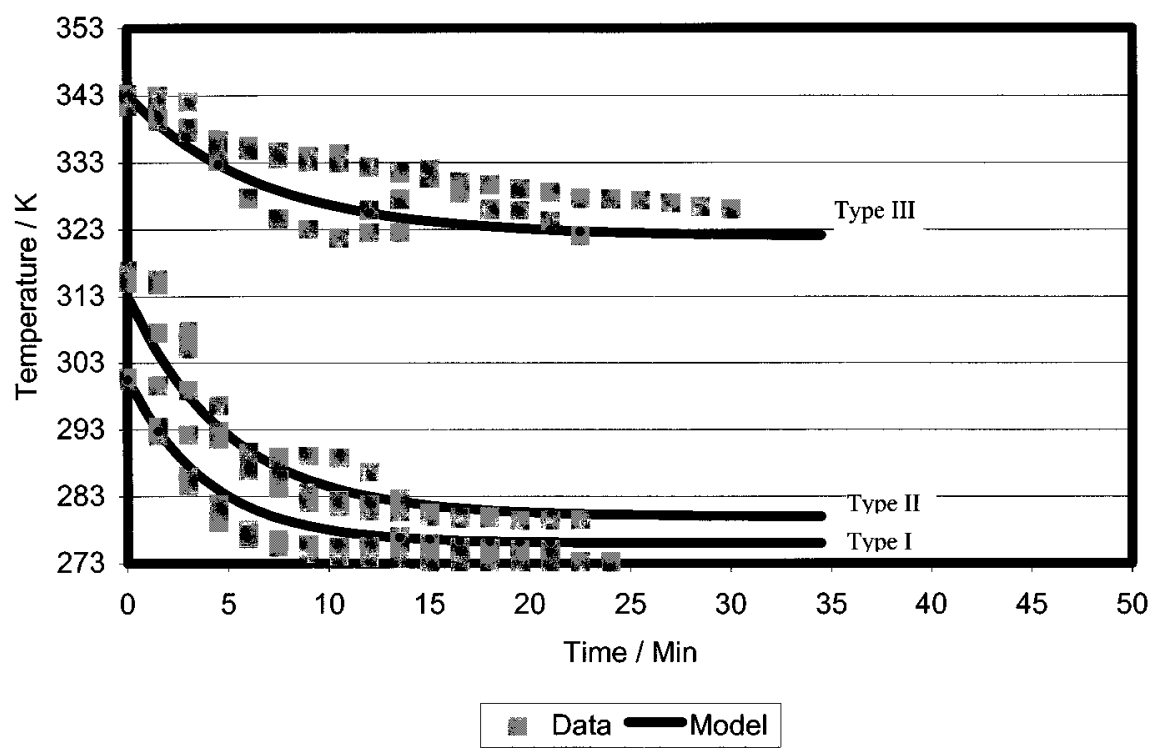

Fig. 4. Cooling of connectors during vehicle travel.

TABLE III

Model Coefficients of TeMPerature Drop Due to Vechile Movement

\begin{tabular}{l|c|c|c|c|c}
\hline Type & $\mathbf{T}_{\mathbf{a m b}}$ & $\Delta \mathbf{T}_{\mathbf{I}}$ & $\Delta \mathbf{T}_{\mathbf{h}}$ & $\mathbf{K}_{\mathrm{cv}}$ & $\mathbf{r}$ \\
& $/ \mathbf{K}$ & $/ \mathbf{K}$ & $/ \mathbf{K}$ & $/ \mathbf{m i n}^{-1}$ & \\
\hline $\mathbf{I}$ & 276 & 0 & 24.5 & 0.25 & 0.992 \\
\hline II & 276 & 4 & 37 & 0.20 & 0.980 \\
\hline III & 276 & 46 & 67 & 0.15 & 0.955 \\
\hline
\end{tabular}

including any further parameter except $\Delta T_{l}$. The experimental data showed that the connectors cooled quicker when the vehicle was moving, due to air flowing around the components. Equation (4) is used to model the experimental data using values from Table III

$$
T_{c}(t)=T_{a m b}+\Delta T_{l}+\left(\Delta T_{h}-\Delta T_{l}\right) \exp \left(-K_{c v} t\right)
$$

Fig. 4 shows plots of experimental data and the model of cooling to $\Delta T_{l}$ for each connector type. The empirical model for all scenarios uses $T_{a m b}, \Delta T_{h}$ and $\Delta T_{l}$ parameters taken from experimental data acquired in the United Kingdom.

\section{DISCUSSION}

\section{A. Maximum Temperature}

Table IV summarizes the maximum temperature attained by the sample connector types studied, $\left(T_{a m b}+\Delta T_{h}\right)^{\circ} \mathrm{C} . \Delta T_{h}$ is the difference between the ambient temperature and the maximum temperature a particular connector type. The proximity of the connector to the engine determines this temperature difference. High temperatures accelerate the physical and chemical processes, which lead to degradation of the connector. Diffusion of copper base material through the contact plating and surface oxidation is known to increase with temperature. Additionally, the change in temperature from ambient to the maximum level
TABLE IV

TEMPERATURE RESULTS

\begin{tabular}{l|c|c|c|c}
\hline Type & $\begin{array}{c}\mathbf{T}_{\mathbf{a m b}}{ }^{+} \\
\Delta \mathbf{T}_{\mathbf{h}} /{ }^{\circ} \mathrm{C}\end{array}$ & $\Delta \mathrm{x}_{\mathbf{1}} / \mu \mathrm{m}$ & $\begin{array}{c}\Delta \mathbf{T}= \\
\Delta \mathbf{T}_{\mathrm{h}_{\mathrm{b}}}-\Delta \mathbf{T}_{\mathbf{l}}\end{array}$ & $\Delta \mathrm{x}_{2} / \mu \mathrm{m}$ \\
\hline I & 24.5 & 4.1 & 24.5 & 4.1 \\
\hline II & 40 & 6.2 & 33 & 5.5 \\
\hline III & 70 & 10.2 & 21 & 3.5 \\
\hline
\end{tabular}

may cause differential thermal expansion, the mechanical expansion, and contraction of components, which can also lead to connector degradation.

\section{B. Temperature Changes}

During travel, the connector temperature varies by $\Delta T$ (between $\Delta T_{h}$ and $\Delta T_{l}$ above ambient) in a complex manner depending upon several parameters. The magnitude of the variation is shown in Table IV for the three types of connector. Assuming that the temperature measured corresponds to the metallic component temperature. Considering a copper component of $1 \mathrm{~cm}$ in length, this would expand by between 3.5-5.5 $\mu \mathrm{m}$ for the temperature variation observed in the three types of connector studied ( $\Delta x_{2}$ in Table IV). This type of temperature change is thought to cause fretting action at the contact interface of the connector [16].

\section{Rate Constants $(K)$}

$K_{h}, K_{c}$, and $K_{c v}$ are rate constants relating to the change in connector temperature from 1) ambient to maximum, 2) maximum to ambient, and 3) maximum to a low level due to vehicle travel, respectively. $K_{h}$ is $0.1 \mathrm{~min}^{-1}$ for all connectors and all heat-up relatively quickly. This is due to the engine being a heat source, which heats to its operating temperature quickly. $K_{c}$ is between $0.004-0.008 \mathrm{~min}^{-1}$; the connectors cool at a 
TABLE V

TIME TO REACH GIVEN TEMPERATURE

\begin{tabular}{c|c|c|c|c|c|c}
\hline Type & \multicolumn{2}{|c|}{ Scenario 1 } & \multicolumn{2}{c|}{ Scenario 2 } & \multicolumn{2}{c}{ Scenario 3 } \\
/min & $t_{50}$ & $t_{98 \%}$ & $t_{50 \%}$ & $t_{98 \%}$ & $t_{50 \%}$ & $t_{98 \%}$ \\
& $\%$ & & & & & \\
\hline I & 6.9 & 39.1 & 86.6 & 489 & 2.8 & 15.6 \\
\hline II & 6.9 & 39.1 & 138 & 782 & 3.5 & 19.6 \\
\hline III & 6.9 & 39.1 & 173 & 978 & 4.6 & 26.1 \\
\hline
\end{tabular}

relatively slow rate compared to heating up. This is due to the engine having a high thermal capacity, which acts to maintain the connector temperature for a longer period. In addition those connectors which are closer to the engine cool slower (Type III, $K_{c}=0.004 \mathrm{~min}^{-1}$ ) than those furthest from the engine (Type I, $K_{c}=0.008 \mathrm{~min}^{-1}$ ). During travel the connectors cool quicker than they are heated, $K_{c v}$ is between 0.15 and $0.25 \mathrm{~min}^{-1}$. This is due to airflow circulating around the connectors. Type III connectors had a higher degree of shielding from the airflow than Type II. Type I had the least shielding and thus cooled at the quickest rate.

The experimental data showed that there were multiple rates of temperature change depending upon the operating scenario. Table V summaries the time taken for connector types to reach particular temperature levels calculated from the empirical model. The model shows that it takes $40 \mathrm{~min}$ to achieve at least $98 \%\left(t_{98 \%}\right)$ of the high operating temperature when the vehicle is stationary. This applies to all three types of connector with a rate constant $K_{h}=0.1 \mathrm{~min}^{-1}$. These have a half-life, $t_{50 \%}=6.9 \mathrm{~min}$. The $t_{98 \%}$ and $t_{50 \%}$ are calculated from the model by (5),

$$
t_{98 \%}=\frac{\ln (50)}{K}, \quad t_{50 \%}=\frac{\ln (2)}{K} .
$$

The rate of cooling of the different types of connector when the vehicle is stationary varies (see $K_{c}$ in Table I) but takes in excess of eight hours to reach $98 \%$ of cooling to ambient temperature (see Table V). When the vehicle is in motion it takes between $15-26$ min to reach $98 \%$ of the low operating temperature.

The temperature profile of a connector is expected to influence its degradation mechanisms. High temperatures increase physical and chemical processes, and temperature changes result in differential thermal expansion of components.

\section{Degradation Mechanisms}

Degradation mechanisms can be divided into three main types: 1) chemical, 2) mechanical, and 3) physical, plus a further type caused by combining mechanisms [2]. High temperature stress and temperature change stress, have an influence on all these mechanisms. Temperatures influence both chemical and physical processes in an Arrhenius manner with increased rate of reaction related to increased temperature [14]. Additionally, temperature changes in a body cause mechanical stress due to differential thermal expansion and contraction of component parts.

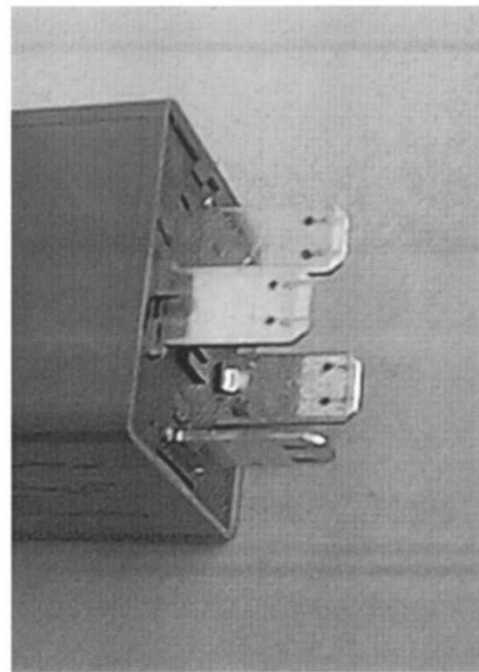

Fig. 5. Fretting corrosion on terminals.

Fretting corrosion is a degradation mechanism, which arises from the combined effect of mechanical micromovement at an interface and corrosion of that surface [16], [17]. Differential thermal expansion and contraction is thought to be the major contributor to low frequency micromovements of contacting interfaces. Tin-based contact systems can be particularly prone to this resulting in high resistance values across the connector.

To investigate connector degradation mechanism, a study has been undertaken using wire harnesses from life tested vehicles. The objective is to gain a better understanding of the influence of environmental stresses on connector degradation.

\section{FIELD STUdy OF CONNECTORS}

To investigate the degradation of connectors in service, a range of life tested wire-harnesses have been studied.

\section{A. Contact Interface}

1) Unsealed Low Temperature Connector: The terminals of unsealed connector sockets and relay modules have been examined. These were tin plated and specified for the low temperature regime. Evidence for fretting corrosion at the contact interface was clearly seen on the samples and is exemplified in Fig. 5. This shows the four terminals of the relay module. The dark spots are the areas of contact, which are covered in small pieces of debris [2] shown more clearly in scanning electron microscopy. Fig. 6(a) is a scanning electron micrograph showing an example of one of these areas. A closer examination of the scar showed debris around the periphery of the scar, an indicator of fretting corrosion. Fig. 6(b) is a copper elemental map of the area and shows high levels of copper in the middle of the wear scar. This indicates that the top plate has undergone wear revealing the copper base material an indicator of fretting.

2) Sealed High Temperature Connector: The connectors studied had tin plated and gold plated terminals. Evidence of fretting corrosion was seen on the tin plated samples as illustrated in Fig. 7. This shows a scanning electron micrograph of the contact area which shows debris scattered around the periphery of the contact area. Fig. 7(b) is a copper elemental 

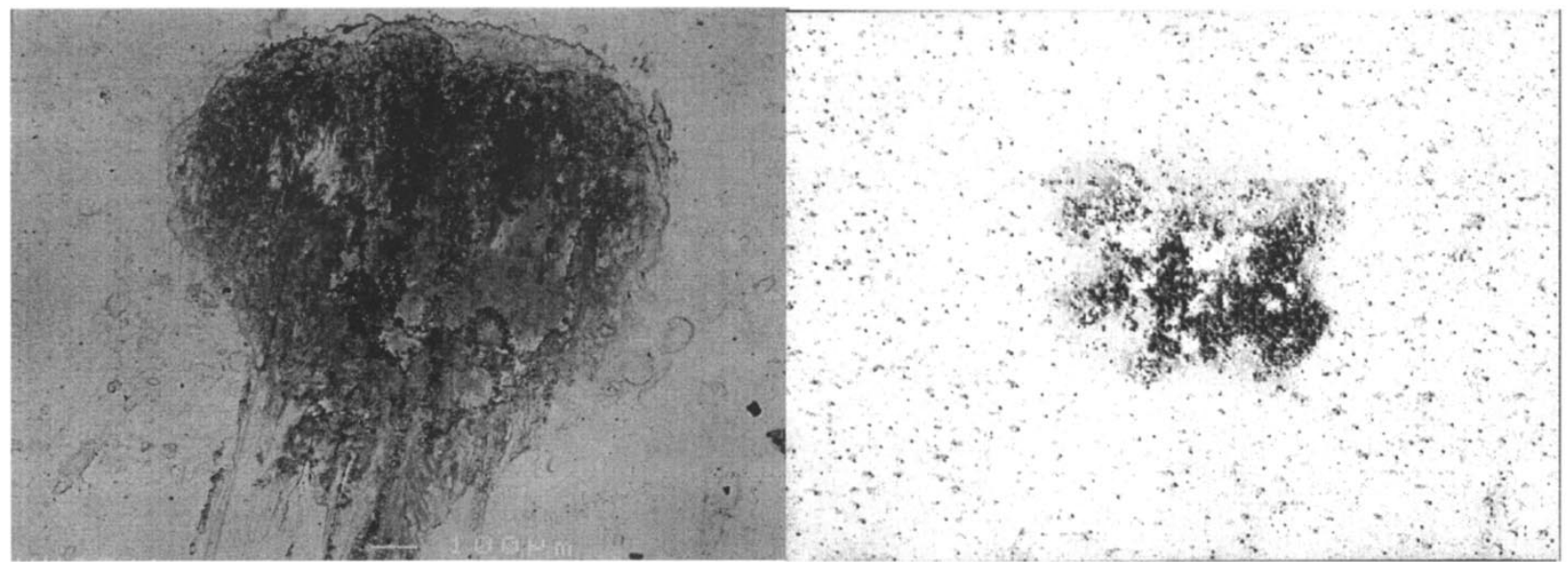

Fig. 6. (a) SEM and (b) Cu analysis of contact area.
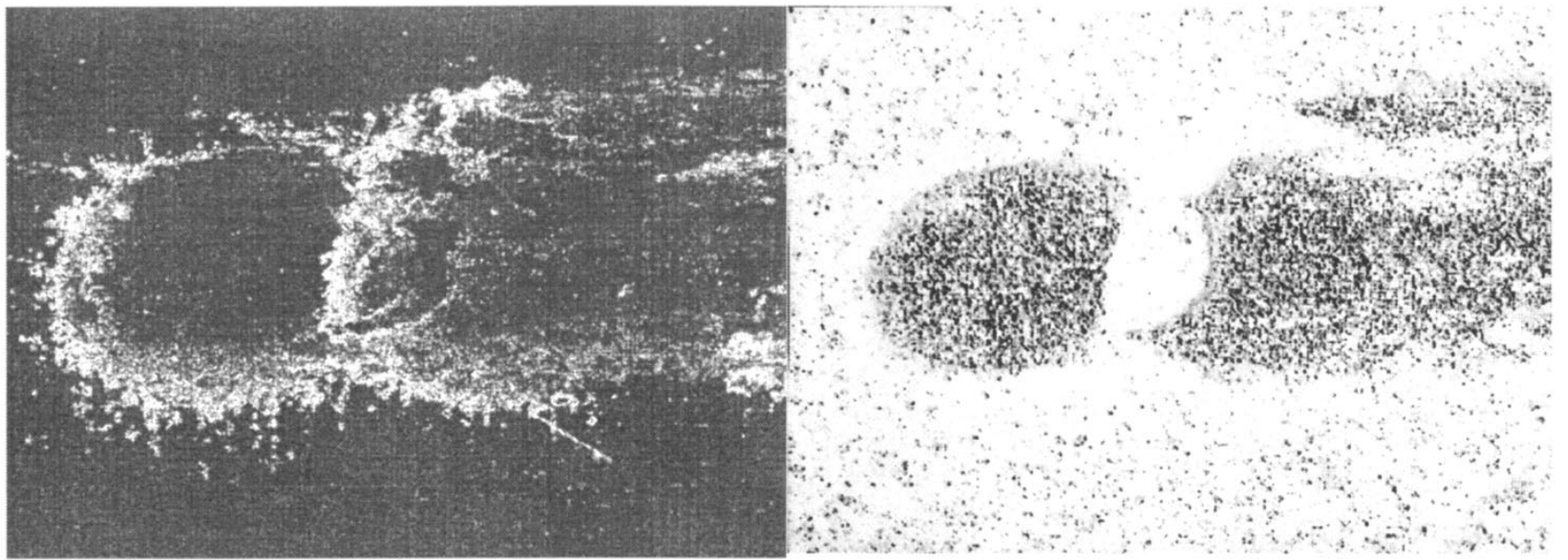

Fig. 7. (a) SEM and (b) $\mathrm{Cu}$ analysis of contact area.

map of this area showing that the top plate has undergone wear by reveal the copper base material. Gold plated terminal showed minimal evidence of wear and corrosion. It was difficult to find any wear scar on the surface.

\section{B. Crimp Interface}

A cross section of a socket crimp from an unsealed low temperature connector is shown in Fig. 8. This is an example of one of the unsatisfactory crimp joint investigated on the harnesses. Voids between conducting strands of the wire and the crimp wall can be seen along with over-folding of the crimp arms. This indicates a poorly formed crimp during the manufacturing process. However, no evidence was found of crimp degradation.

\section{Resistance Measurements}

The electrical resistance across a connector will include the crimp contact resistance, the resistance across the bulk material used in the terminals and the resistance across the contact interface. Increases in resistance due to degradation usually occur across the contact interface and across the crimp interface.

Resistance measurements were taken across twenty-four unsealed low temperature connector terminals from wiring harnesses of vehicles, which had undergone road testing.

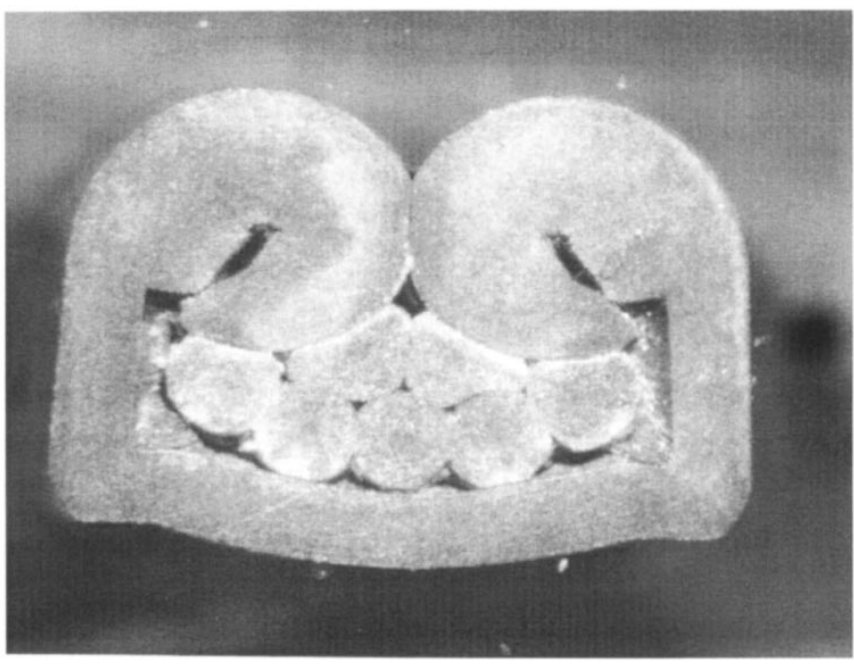

Fig. 8. Cross-section of crimp.

Two dry circuit measurements were taken across, the contact interface and crimp interface together, and the contact interface only. Fig. 9 shows a range of resistance values measured across 24 sample terminals. Three plots are shown: 1) a measurement across the whole connector terminal which includes the contact 


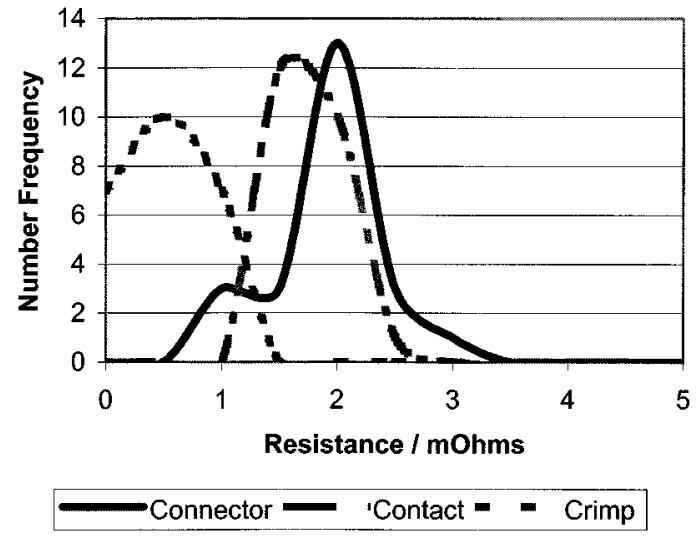

Fig. 9. Resistance measurement of connector terminals.

interface and crimp, 2) a measurement across the contact interface, and 3) by subtracting the previous two, a value is calculated which includes the resistance value across the crimp interface.

Fig. 9 shows that the crimp interface gives a contact resistance value lower than the contact interface between the two terminals. This would be expected since that the crimp contacting force would be higher than the contact normal force supplied by a spring mechanism. The results presented in Fig. 9 show low connector resistance even though both the crimp interfaces and the contact interface are far from satisfactory. A feature of fretting corrosion is that the interface can self-heal by pushing resistive debris away from the metal-to-metal contact areas, thus maintaining a low resistance value. However, intermittent high values can occur.

\section{Discussion of Field Data Results}

The range of temperature variation seen by the automotive connector shown in this paper could be expected to lead to fretting behavior. The data shown in Fig. 1 over a 24-h period can be simplified into approximately 10 cycles of heating and cooling. It has been demonstrated elsewhere that tin based connectors with a single rider can demonstrate a contact resistance failure after as few as 100 cycles, [18]. In most cases commercial connectors use a minimum of two connecting surfaces, and in this case failure has not been detected after 2000 cycles, [2]. It is proposed that the difference between the two results is because of the self-healing affect and that to get a failure with two surfaces both contacts should reach failure at the same time. The results shown in this paper for the field-tested connectors show no significant contact resistance failure, but show wear scars. Either temperature cycling or vibrations can produce the wear scars identified in the study.

\section{CONCLUSIONS}

The results presented are an initial study of connector performance in a vehicle. This paper presents field data on the temperature cycling of connectors. It shows that the cycling process is a complex interaction of the driving conditions and the environment. In some situations rapid changes in the temperature have been observed, the monitoring of which has been limited by the sample rate used. There is a need to improve the sample rate. Measurements currently being made use a 10-s sampling interval. The results presented are for the temperature on the connector housing.

An empirical model of temperature behavior for three types of connector is presented based on real environmental data obtained from the road-tested vehicles. The model simulates heating and cooling of the connector when the vehicle is stationary and moving. When the vehicle is traveling the behavior is complex but is bounded by an upper and lower temperature limit.

High temperatures accelerate chemical and physical processes leading to degradation of the connector. Temperature changes cause differential thermal expansion and contraction, which lead to fretting corrosion. Both sealed and unsealed tin plated connector terminals, which had undergone road testing, showed evidence of fretting corrosion. Debris and a wear scar were found at the contact interface on sample connectors. However, contact resistance values were within a satisfactory level when measured in the laboratory before the terminals were separated.

\section{ACKNOWLEDGMENT}

The authors would like to thank G. Bolgar and his team, Jaguar Cars Ltd., for the access to vehicles for the acquisition of environmental data and C. Chalk for resistance measurements of harness connectors.

\section{REFERENCES}

[1] G. Bolger, "The selection of automotive connectors," Senior thesis, Coventry Univ., U.K., 1997.

[2] J. Swingler and J. W. McBride, "The synergistic relationship of stresses in the automotive connector," in Proc. 19th Int. Conf. Electric Contact Phenom., Nuremburg, Germany, 1998, pp. 141-145.

[3] I. O. Wallinder and P. Eriksson, "Characterization of the corrosivity of an automotive environment," in Proc. 19th Int. Conf. Electric Contact Phenom., Nuremburg, Germany, 1998, pp. 157-161.

[4] W. H. Abbott, "Time distribution of intermittents verses contact resistance for tin-tin connector interfaces during low amplitude motion," IEEE Trans. Comp., Hybrids, Manufact. Technol., vol. CHMT-7, pp. 107-111, Jan. 1984.

[5] S. R. Murrel and S. L. McCarthy, "Intermittence detection in fretting corrosion studies of electrical contacts," in Proc. IEEE 43rd Holm Conf. Electrical Contacts, 1997, pp. 1-6.

[6] J. Swingler, "Degradation of electrical contacts under low frequency fretting conditions," Ph.D. dissertation, Loughborough Univ., U.K., 1994.

[7] W. H. Abbott, "Materials, environment, motion and electrical contacts," in Proc. IEEE Holm Conf. Electrical Contacts, 1989, pp. 3-11.

[8] W. Abbott, "Effects of test procedures and sequences on the performance of tin-plated connectors," in Proc. IEEE 39th Holm Conf. Electrical Contacts, 1993, pp. 191-204.

[9] R. D. Malucci, "Accelerated testing of tin plated copper alloy contacts," in Proc. IEEE Holm Conf. Electrical Contacts, 1997, pp. 138-151.

[10] R. S. Mroczkowski, "Concerning reliability modeling of connectors," in Proc. IEEE Holm Conf. Electrical Contacts, 1998, pp. 57-68.

[11] _ , "Concerning estimating connector reliability: Laboratory simulations and reliability modeling," in 19th Int. Conf. Electric Contact Phenom., Nuremburg, Germany, 1998, pp. 135-139.

[12] USCAR/EWCAP Standard for Automotive Electrical Connection Systems, 1994.

[13] N. Saka, M. J. Liou, and N. P. Suh, "The role of tribology in electrical contact phenomena," Wear, vol. 100, pp. 77-105, 1984.

[14] C. Maul, J. Swingler, and J. W. McBride, "Monitoring the connector environment in automotive systems," in Proc. IEE Symp. Automotive Electron. Standards, London, U.K., Nov. 30, 1999.

[15] P. W. Atkins, Physical Chemistry, 3rd ed. Oxford, U.K.: Oxford Univ. Press, 1987. 
[16] R. B. Waterhouse, Fretting Corrosion. New York: Pergamom, 1972.

[17] M. Antler, "Fretting corrosion of solder-coated electrical contacts," IEEE Trans. Comp., Hybrids, Manufact. Technol., vol. CHMT-7, pp. 129-138, Jan. 1984.

[18] N. A. Stennett and J. Swingler, "The effect of power on low frequency fretting corrosion," in Proc. 39th IEEE Holm Conf. Electrical Contacts, Pittsburgh, PA, USA, Sept. 1993, pp. 205-210.

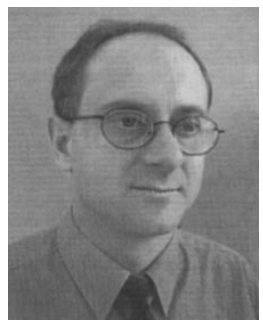

Jonathan Swingler received the Joint Honors degree in physics and chemistry from the University of Keele, U.K., in 1990 and the Ph.D. degree in electrical contact science and technology from Loughborough University, U.K., in 1994. His Ph.D. research was primarily concerned with degradation processes at connector contact surfaces and the influence of lubricants in imporving contact performance.

He later moved to the University of Southampton, U.K., to investigate contact materials for medium duty switching, arc modeling, hybrid switching, constriction resistance measurement, and connector reliability. He is currently a Senior Research Fellow at the University of Southampton, U.K.

Dr. Swingler is a Chartered Physicist, member of the Institute of Physics, and an associate member of the Institute of Electrical Engineers.
John McBride (M'99) received the M.S. degree in aeronautical engineering from the University of Southampton, Southampton, U.K., in 1978 and the $\mathrm{Ph} . \mathrm{D}$. degree in electrical contact phenomena from Plymouth University, U.K., in 1986.

From 1985 to 1987, he lectured in the Mechanical Engineering Department, Plymouth University, and, since 1987, has been a Lecturer, Senior Lecturer, and a Reader in instrumentation and measurement in the School of Engineering Science and the Electrical Engineering Department, University of Southampton. His main research interests include electrical contacts, metrology, and instrumentation.

He is a Chartered Engineer and Chair of the Electro-Mechanical Research Group.

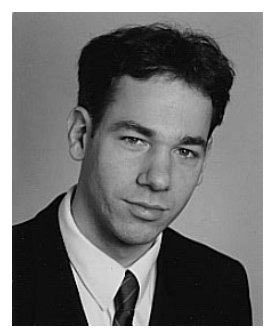

Christian Maul received the Dipl.-Ing. degree in mechanical engineering from the Technical University of Braunschweig, Germany, in 1997 and is currently pursuing the Ph.D. degree at the University of Southampton, Southampton, U.K.

His work involves fundamental and applied research in automotive connectors. 